\title{
Editorial
}

\section{Physics of Quark Gluon Plasma: An Update and the Status Report}

\author{
Jan E. Alam, ${ }^{1}$ Edward Sarkisyan-Grinbaum, ${ }^{2,3}$ and Subrata Bhattacharyya ${ }^{4}$ \\ ${ }^{1}$ Theoretical Physics Division, Variable Energy Cyclotron Centre, Kolkata 700064, India \\ ${ }^{2}$ PH Department, CERN, 1211 Geneva 23, Switzerland \\ ${ }^{3}$ Department of Physics, The University of Texas at Arlington, Arlington, TX 76019, USA \\ ${ }^{4}$ Physics \& Applied Mathematics Unit, Indian Statistical Institute (ISI), Kolkata 700108, India
}

Correspondence should be addressed to Jan E. Alam; jane@vecc.gov.in

Received 24 February 2014; Accepted 24 February 2014; Published 9 June 2014

Copyright (C) 2014 Jan E. Alam et al. This is an open access article distributed under the Creative Commons Attribution License, which permits unrestricted use, distribution, and reproduction in any medium, provided the original work is properly cited. The publication of this article was funded by SCOAP $^{3}$.

The microsecond old universe was too hot for the nucleons to survive as bound states of quarks. When the temperature of the universe fell below $\sim 10^{13} \mathrm{~K}$ as a result of cooling due to expansion, the quarks got confined inside the nucleons to reside there ever after-making them inaccessible to any direct observation in isolation. Natural objects like the core of the compact astrophysical objects may also contain deconfined quark matter. Therefore, creation of a deconfined phase of thermal quarks and gluons will be very important for understanding the evolution of the early universe and the physics of compact astrophysical objects like neutron star. Calculations based on lattice QCD indicate that hadronic matter at high temperatures and densities undergoes a phase transition to a thermalized system of deconfined quarks and gluons, called quark gluon plasma (QGP). It is expected that such high densities and temperatures may be achieved by colliding two nuclei at RHIC, LHC, or FAIR energies. The present volume addresses some of the experimental and theoretical issues related to quark-hadron phase transition in the relativistic heavy ion collisions by the world experts in the field. On the experimental sides it contains results from CERN's Super Proton Synchrotron (SPS) and Large Hadron Collider (LHC) and Brookhaven National Laboratory's Relativistic Heavy Ion Collider (RHIC). Comparison of experimental results from RHIC and LHC is extremely useful to gain insights into the properties of the matter formed in these experiments. The present volume offers a review on this subject. On the theoretical side the present volume contains papers on the space-time evolution of the system formed in heavy ion collisions including the effects of viscosities through hydrodynamic equations, issues of equilibration, electromagnetic and heavy quark probes of QGP, quarkonia suppression in QGP, ridge, and transverse correlations. Recent progress in the femtoscopy and particle production in strong electromagnetic fields in heavy ion collisions is also addressed adequately. In summary, the present volume brings out recent developments on almost all the relevant issues of heavy ion collisions like creation, evolution, and signals of QGP. We are sure that the volume will be useful for the current practitioners and young new entrants to this exciting field of research.

\section{Acknowledgment}

We sincerely thank all the authors who contributed to this volume.

Jan E. Alam

Edward Sarkisyan-Grinbaum Subrata Bhattacharyya 

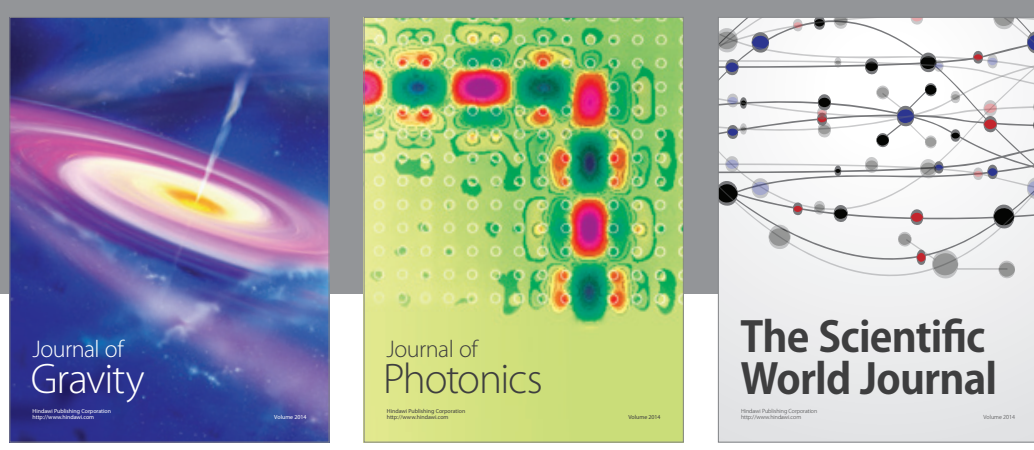

The Scientific World Journal
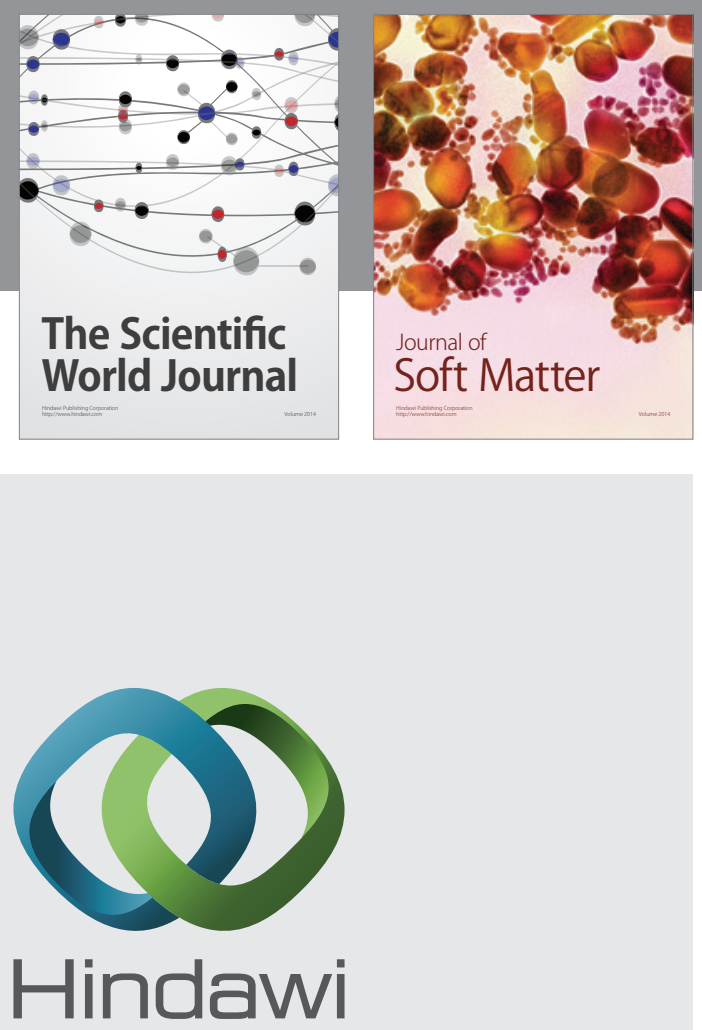

Submit your manuscripts at

http://www.hindawi.com

nternational Journal of

Statistical Mechanics
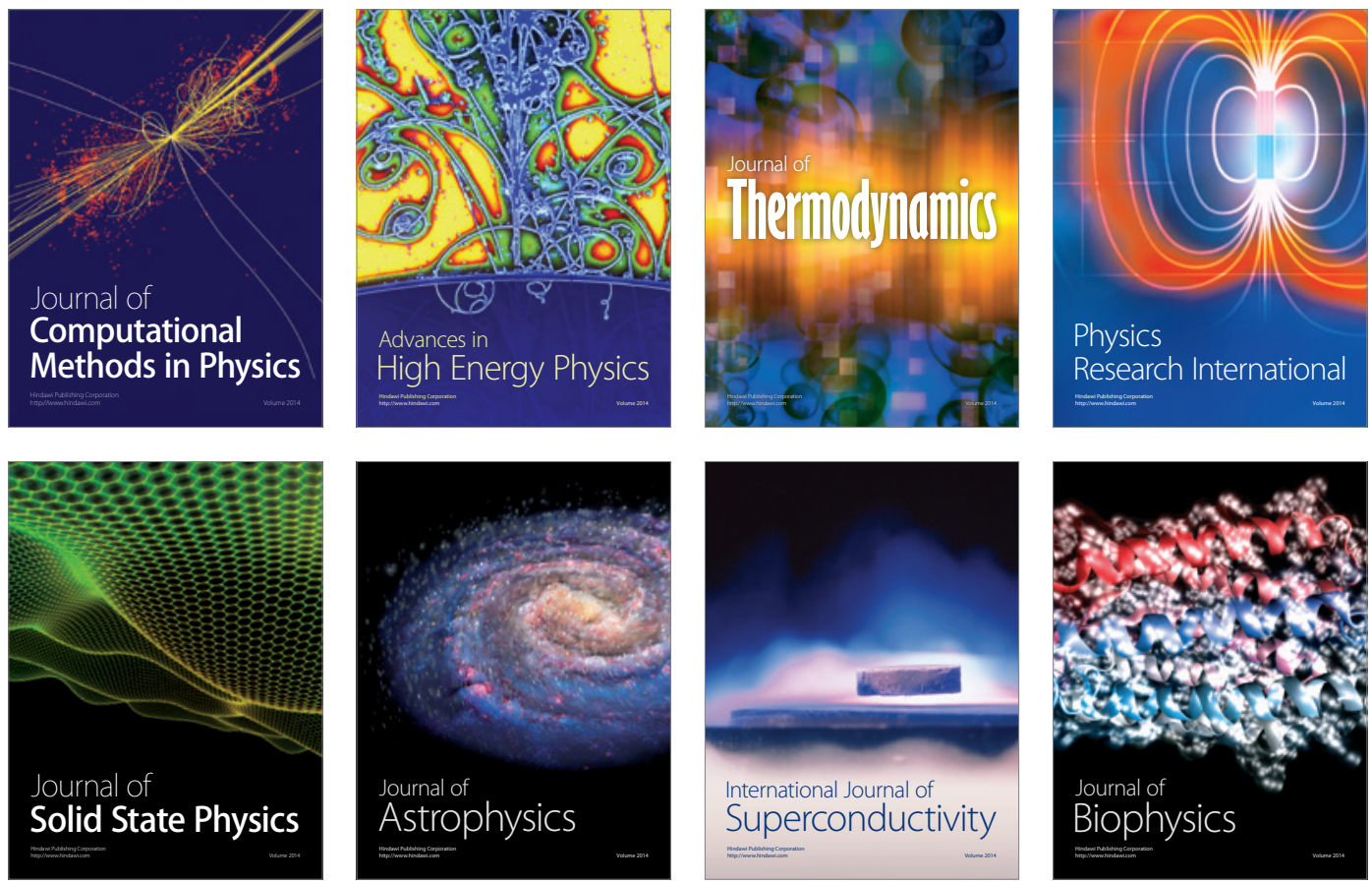
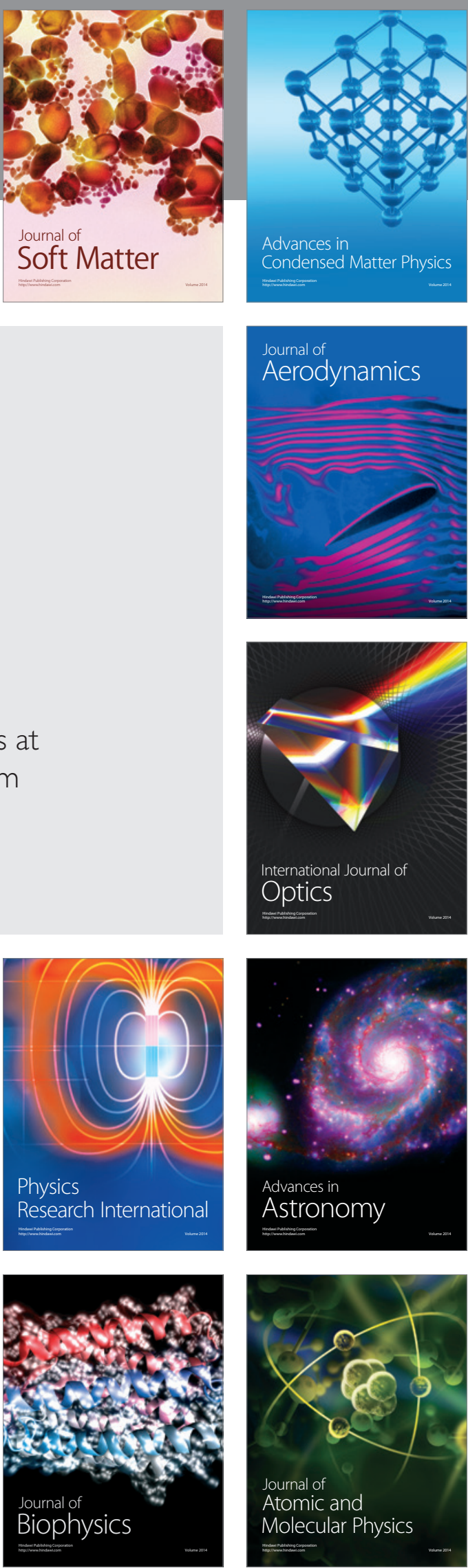